\title{
Laboratory Evaluation of Oviposition Behavior of Field Collected Aedes Mosquitoes
}

\author{
Subrat K. Panigrahi, Tapan K. Barik, Satyabrata Mohanty, and Niraj Kanti Tripathy \\ Post Graduate Department of Zoology, Berhampur University, Berhampur, Odisha 760007, India \\ Correspondence should be addressed to Niraj Kanti Tripathy; nirajtripathy@rediffmail.com
}

Received 24 January 2014; Revised 27 May 2014; Accepted 4 June 2014; Published 1 July 2014

Academic Editor: José A. Martinez-Ibarra

Copyright (C) 2014 Subrat K. Panigrahi et al. This is an open access article distributed under the Creative Commons Attribution License, which permits unrestricted use, distribution, and reproduction in any medium, provided the original work is properly cited.

Wild female Aedes aegypti and Ae. albopictus were allowed to lay eggs in (i) ovitraps with different concentrations of $\mathrm{NaCl}$, (ii) different coloured ovistrips, (iii) water from different sources, (iv) larva holding water, and different sized ovitraps for oviposition preference. Oviposition cycle was also studied in different photoperiod regimens. The number of eggs laid was observed to gradually decrease with increase in $\mathrm{NaCl}$ concentration in both the species. Experiments were conducted to determine egg laying preference for any specific colour of the ovistrip and black ovistrip was found to be most preferred by both the species. For oviposition preference, eight water samples collected from different sources were used and it was observed that the maximum number of eggs was laid in ovitraps containing distilled water followed by tap water. In addition, Aedes mosquitoes laid more number of eggs in ovitraps containing larval holding water than ovitraps containing distilled water. Further, both the species did not lay any egg in the smallest used ovitrap although the number of eggs was maximally deposited in the largest ovitrap used. In the present studies, both the Aedes species laid the maximum number of eggs in the 4th quarter of the light period with normal $12 \mathrm{~h}$ light and dark phases (LD 12:12).

\section{Introduction}

Globally, Aedes aegypti and Aedes albopictus are the principal mosquito vectors of dengue and dengue hemorrhagic fever viruses [1]. Larval stages of both mosquitoes inhabit containers in residential landscapes. Selection of an oviposition site by the female mosquitoes is one of the most important behavioural components of their survival [2]. A female mosquito chooses oviposition sites by a combination of visual and chemical cues. Ovipositing mosquitoes taste the water in a potential oviposition site to detect chemical cues [2]. Further, mosquitoes may also select oviposition sites based on the availability of larval food [3]. Normally a female does not lay her entire batch of eggs in one location but rather distributes them in multiple water-filled containers, a behaviour called skip-oviposition [4]. This behaviour increases the distribution of eggs over a larger area and may be increased by the tendency of gravid females to avoid ovipositing in sites where eggs of conspecific females have been laid [5-7]. Gravid Aedes females lay their eggs in water-filled containers. However, very few cases have been examined to explain the role of container shape and size in oviposition site selection. Aedes mosquitoes are usually day active mosquitoes and might be relying more on optical cues like the contrast between dark container openings for selection of resting and oviposition sites than night active mosquito species [8]. Further, Aedes mosquitoes breed in different types of household containers including different coloured flower pots. Colton et al. [4] reported that Aedes mosquitoes lay maximum number of eggs on black ovistrips. Further, female mosquitoes normally avoid oviposition where interspecific competitors are present [3]. It has been earlier reported that larvae of many mosquito species develop well in salt water and such species prefer higher salt content of the medium for oviposition [9]. The mosquito life cycle is disturbed if oviposition is prevented and, thereby, population growth can be reduced. Hence, understanding the oviposition behavior of mosquitoes may not only give a new insight about their life history, but also lead to more refined dengue surveillance and control practices. 
In the present study, in order to find out oviposition preference of the mosquito vectors, if any, the gravid females were allowed to lay eggs in ovitraps with different concentrations of $\mathrm{NaCl}$, on different coloured ovistrips, in water from different sources, in larva holding water, and in different sized ovitraps. The oviposition cycle of the vectors was also studied in different photoperiod regimens like normal light dark, reversed light dark, and continuous light. The results obtained are summarized in the present study for further use of the data to formulate strategies for the control of Aedes mosquito vectors.

\section{Materials and Methods}

2.1. Collection and Mass Rearing of Aedes Mosquitoes. Both immature and adult stages of Aedes aegypti and Ae. albopictus were collected from different localities in and around Berhampur city, Odisha State of India, and were transported to the laboratory for mass rearing. The mosquitoes were identified under a binocular stereo zoom microscope in the laboratory as per the identification keys. The mosquito colony was maintained at $26 \pm 1^{\circ} \mathrm{C}$ and at a relative humidity of $\sim 75 \%$ under a photoperiod of $14: 10 \mathrm{~h} \mathrm{~L}: \mathrm{D}$. Larvae were fed a $2: 1$ mixture of liver powder: baker's yeast [8]. Adults were kept in $30 \times 30 \times 30 \mathrm{~cm}$ Plexiglas cages fitted with cotton surgical stocking tops and maintained on a $10 \%$ sucrose solution provided ad libitum.

For all the experiments pertaining to the selection of oviposition medium and preference for oviposition, the laboratory-bred colonies of Ae. aegypti and Ae. albopictus collected from local population were used.

2.2. Effect of $\mathrm{NaCl}$ Concentration on Oviposition. Graded series of $\mathrm{NaCl}$ concentrations (of analytical grade) in dechlorinated tap water by volumetric dilution were tested for oviposition preference of Aedes mosquitoes. The tested concentrations of $\mathrm{NaCl}$ ranged from $0.25 \%$ to $2.0 \%$. Dechlorinated tap water was used as the control medium for comparison of the results.

Five-six-day-old females were given a blood meal (vide supra) and transferred into cages $\left(24^{\prime \prime} \times 24^{\prime \prime} \times 24^{\prime \prime}\right)$ in batches of 100 females per cage. After $48 \mathrm{~h}$ of the blood meal, ovitraps were introduced into the cages containing dechlorinated water which served as control and variable concentrations of $\mathrm{NaCl}$. No definite pattern of arrangement of ovitraps was followed in the cages. Eggs were collected for a period of $24 \mathrm{~h}$ (starting from $6 \mathrm{pm}$ to the $6 \mathrm{pm}$ of next day). The experiment was repeated 5 times and the data obtained were recorded for further statistical analysis.

2.3. Oviposition in Response to Colour of Ovistrip. In order to find out if Aedes mosquitoes have a colour preference for oviposition, the ovitraps were covered with different coloured velvet papers (black, red, yellow, green, blue, and white) with the ability to soak water were used in the present study. Ovitraps were covered with coloured velvet papers dipped half in the water inside to provide moist surface for egg laid by the gravid females and kept arbitrarily inside the cages without any specific sequence of arrangement.

Five-six-day-old gravid females, after a blood meal, in groups of 100 females per batch, were introduced into the cages for egg collection. The eggs were collected for a period of $24 \mathrm{~h}$ when the insectary was kept lighted for $12 \mathrm{~h}$ to facilitate the females to distinguish between different colours of the ovistrips. The experiment was repeated five times and the data so collected were subjected to statistical evaluation. Similar experiments were conducted in dark to determine if the mosquitoes have any visual perception.

2.4. Oviposition in Response to Water from Different Sources. Experiments were conducted in cages $\left(24^{\prime \prime} \times 24^{\prime \prime} \times 24^{\prime \prime}\right)$ under controlled environmental conditions. Five-six-day-old gravid females of both species, after a blood meal, were grouped into 100 mosquitoes per cage separately. After $48 \mathrm{~h}$ of blood meal, circular ovitraps of $4^{\prime \prime}$ diameter half-filled with water from different natural sources like distilled water, tap water, pond, sea, well, paddy field, drain, and ditches were introduced into the cages. The ovitraps were internally coated with filter paper up to half the water level to act as the moistened surface for egg laying by the female Aedes mosquitoes. The ovitraps were introduced into the cages at $6 \mathrm{pm}$ and removed the next day at $6 \mathrm{pm}$. The number of eggs laid by both species of Aedes females was counted with a hand lens. The ovitraps were kept arbitrarily without any definite pattern of arrangement inside the cage. Five replicate experiments were conducted and the data obtained were recorded for statistical analysis.

2.5. Oviposition Preference in Larva Holding Water. In this experiment, in order to find out if the gravid females have any preference for egg laying in water holding larvae over water without any larvae, one hundred blood-fed female Aedes mosquitoes per cage were kept in cages of dimensions of $24^{\prime \prime} \times 24^{\prime \prime} \times 24^{\prime \prime}$. Ovitraps containing dechlorinated tap water containing at least 10-20 larvae were introduced into the cages at $6 \mathrm{pm}$ and were kept as such for $24 \mathrm{~h}$. The experiment was repeated 5 times and the numbers of eggs counted in each experiment were recorded. The data were subjected to statistical analysis.

2.6. Container Size for Oviposition Preference. Blood-fed gravid females, 100 per batch, were transferred into cages. In order to find out the preference, if any, of the mosquitoes for egg laying with respect to the size of ovitrap, black ovitraps of different diameters ranging from $1.5 \mathrm{~cm}$ to $8 \mathrm{~cm}$ were kept inside the cages without any definite arrangement or sequence. Egg collections were made for $24 \mathrm{~h}$ starting from $6 \mathrm{pm}$ to $6 \mathrm{pm}$ of the next day. The number of eggs laid in each ovitrap was counted with a hand lens. The experiment was repeated 5 times and the data obtained were subjected to further statistical calculations.

2.7. Oviposition Cycle. As in the above experiment, five-sixday-old gravid and blood-fed female Aedes mosquitoes, 100 per batch, were allowed to lay eggs in ovitraps half-filled with dechlorinated tap water internally lined with filter paper in 
cages of $24^{\prime \prime} \times 24^{\prime \prime} \times 24^{\prime \prime}$ dimension. The number of eggs laid was checked at intervals of $3 \mathrm{~h}$. The ovitraps were replaced every time. Observations were made for three consecutive days. The following three different types of photoperiods were tested for each species of Aedes mosquito following Suleman and Shirin [10]:

(a) normal (light-day) photoperiod (LD $12: 12$ );

(b) reversed (dark-light) photoperiod (DL 12:12);

(c) continuous light photoperiod (24 LL).

In the experiments (a), (b), and (c), the mosquitoes lived in normal light conditions. Fluorescent tubes were used as the light source in the insectary. During the dark period, inspections were made with a red light torch, since mosquitoes are supposed to be blind to red light [11]. The experiments were repeated 5 times and data obtained were analyzed statistically.

\section{Results}

In order to understand the oviposition behaviour of field collected Aedes mosquitoes, experiments were conducted in response to varied $\mathrm{NaCl}$ concentration, choice of ovistrip colour, choice of oviposition with water from different sources and larval holding water, oviposition preference in response to size of ovitrap, and oviposition cycle at different photoperiod regimens in the present investigation.

3.1. Choice of Oviposition Medium with Varied NaCl Concentration. In order to test the preference for salinity of the water in the breeding site, ovitraps containing different concentrations of $\mathrm{NaCl}$ solutions ranging from $0.25 \%$ up to $2.0 \%$ were tested. Ovitrap containing distilled water was used as the control. It was observed that Ae. aegypti females laid maximum number of eggs at $0.25 \%$ of $\mathrm{NaCl}$ while minimum numbers of eggs were laid at $2.0 \% \mathrm{NaCl}(1.8 \pm 1.79)$. In the control ovitrap which contained distilled water, the number of eggs laid was slightly less than the number in $0.25 \% \mathrm{NaCl}$ (Table 1).

With the increase in the concentration of $\mathrm{NaCl}$ in the oviposition medium the number of eggs laid gradually reduced exhibiting a negative correlation between the number of eggs laid and the concentration of sodium chloride in the oviposition medium. In case of Ae. albopictus, while no eggs were laid by the females in the ovitrap containing $2.0 \%$ $\mathrm{NaCl}$ solution, they had laid maximum number of eggs in the ovitrap containing distilled water that served as the control. Here, too, the number of eggs laid gradually decreased with an increase in the concentration of $\mathrm{NaCl}$ in the oviposition medium (Table 1).

3.2. Choice of Ovistrip Colour for Oviposition. Six different coloured ovistrips, namely, black, red, yellow, blue, green, and white, were chosen in the present experiment for collection of eggs to determine if the Aedes mosquitoes under study have preference for any specific colour of the ovistrip for egg laying. The experiment was undertaken both in light and
TABLE 1: Effect of $\mathrm{NaCl}$ concentrations on oviposition of Aedes mosquitoes.

\begin{tabular}{lcc}
\hline \multirow{2}{*}{$\mathrm{NaCl}$ concentration (\%) } & \multicolumn{2}{c}{ Aedes mosquito species } \\
& Ae. aegypti & Ae. albopictus \\
\hline Distilled water & $371.6 \pm 51.11$ & $291.8 \pm 37.51$ \\
0.25 & $392.2 \pm 70.49$ & $279.8 \pm 34.43$ \\
0.5 & $255.6 \pm 53.16$ & $215.6 \pm 56.72$ \\
0.75 & $115.8 \pm 25.67$ & $183.6 \pm 52.61$ \\
1.0 & $110.2 \pm 37.04$ & $84.2 \pm 42.89$ \\
1.5 & $65.2 \pm 16.69$ & $47.4 \pm 28.89$ \\
2.0 & $1.8 \pm 1.79$ & $0 \pm 0.00$ \\
\hline
\end{tabular}

Mean number of eggs \pm SD deposited in water containing different concentrations of sodium chloride $(\mathrm{NaCl})$ by Aedes mosquitoes.

in dark with a view to find out if the Aedes mosquitoes are able to differentiate between different colours of ovistrips in light. It has been observed that Ae. aegypti females laid a maximum of $426.67 \pm 25.66$ eggs in the ovitrap containing black ovistrip in the dark. The numbers of eggs laid in decreasing order of preference were red, yellow, green, and blue, while no eggs were laid in ovitraps containing white ovistrip. When the Ae aegypti females were allowed to lay eggs in a lighted condition in ovitraps containing the above six different coloured ovistrips, a maximum number of eggs ( $343.33 \pm 51.47)$ were collected on black ovistrip. The decreasing order of preference was red, green, yellow, blue, and white. A minimum of $36.67 \pm 10.41$ eggs were laid on white ovistrip (Table 2).

Similarly, in case of Ae. albopictus, when the females were kept in cages containing ovitraps lined with the above six different coloured ovistrips, a maximum of $446.67 \pm 7.51$ eggs were collected from the black ovistrip and a minimum of $35.00 \pm 15.39$ were recovered from the blue ovistrip in dark. Here, too, no eggs were laid by Ae. albopictus females on white ovistrip in darkness as was the case of Ae. aegypti. Under light condition also the females of this species laid maximum numbers of eggs $(328.33 \pm 2.89)$ on black ovistrip. A minimum of $6.00 \pm 1.73$ eggs were observed on white ovistrip in light condition. The numbers of eggs laid on different coloured ovistrips in decreasing order were red, green, yellow, and blue.

A comparison between the average eggs deposited on different coloured ovistrips in dark and light conditions was made by statistical analysis following $t$-test. There was no statistical difference between the number of eggs laid in dark and the number of eggs laid in light on any particular coloured ovistrip by the females of either species.

3.3. Choice of Oviposition Medium (Water from Different Sources). In order to study the oviposition preference, if any, by the two species of Aedes mosquitoes, eight different types of water samples collected from pond, tap, sea, well, paddy field, drain, ditch, and distilled water were used. Maximum numbers of eggs were laid in ovitraps containing distilled water followed by tap water (Table 3). 
TABLE 2: Oviposition preference for different coloured ovistrips by Aedes mosquitoes.

\begin{tabular}{lccccc}
\hline \multirow{2}{*}{ Serial number } & Colour of ovistrip & \multicolumn{3}{c}{ Number of eggs laid by Aedes mosquitoes } \\
& & Ae. aegypti & Dark & Light & Dalbopictus \\
\hline$(1)$ & Black & $343.33 \pm 51.47$ & $426.67 \pm 25.66$ & $328.33 \pm 2.89$ & $446.67 \pm 7.51$ \\
$(2)$ & Red & $330.00 \pm 17.00$ & $335.33 \pm 28.36$ & $275.67 \pm 17.62$ & $330.67 \pm 20.13$ \\
$(3)$ & Yellow & $94.00 \pm 9.54$ & $169.00 \pm 4.00$ & $104.67 \pm 4.04$ & $164.33 \pm 14.57$ \\
$(4)$ & Green & $103.00 \pm 10.44$ & $136.00 \pm 4.00$ & $121.67 \pm 4.93$ & $136.67 \pm 4.04$ \\
$(5)$ & Blue & $38.67 \pm 2.08$ & $29.67 \pm 1.15$ & $39.00 \pm 16.82$ & $35.00 \pm 15.39$ \\
$(6)$ & White & $36.67 \pm 10.41$ & $0.00 \pm 0.00$ & $6.00 \pm 1.73$ & $0.00 \pm 0.00$ \\
\hline
\end{tabular}

Oviposition preference for different coloured ovistrips by Aedes mosquitoes in light and dark (data are represented as mean \pm SD).

TABLE 3: Oviposition preference in water from different sources by the two species of Aedes mosquito.

\begin{tabular}{lccc}
\hline \multirow{2}{*}{ Serial number } & \multirow{2}{*}{ Types of water } & \multicolumn{2}{c}{ Eggs laid by Aedes mosquitoes } \\
& & Ae.aegypti & Ae. albopictus \\
\hline$(1)$ & Distilled water & $346.33 \pm 38.55$ & $342.67 \pm 67.24$ \\
$(2)$ & Pond & $195.67 \pm 31.09$ & $201.00 \pm 73.65$ \\
$(3)$ & Tap & $288.33 \pm 62.88$ & $300.00 \pm 10.54$ \\
$(4)$ & Sea & $0.00 \pm 0.00$ & $0.00 \pm 0.00$ \\
$(5)$ & Well & $253.67 \pm 42.90$ & $241.67 \pm 73.35$ \\
$(6)$ & Paddy field & $170.00 \pm 16.70$ & $119.33 \pm 31.72$ \\
$(7)$ & Drain & $3.33 \pm 3.51$ & $17.00 \pm 5.00$ \\
$(8)$ & Ditches & $186.67 \pm 18.34$ & $165.00 \pm 49.69$ \\
\hline
\end{tabular}

Mean number of eggs \pm SD deposited in water collected from different sources of water by the two species of Aedes.

No eggs were laid by either species in the ovitraps containing sea water while negligible numbers of eggs were observed in ovitraps containing drain water.

3.4. Choice of Oviposition Medium (Normal and Larval Holding Water). In this experiment, mature/blood-fed Aedes females of both species were allowed to deposit eggs in ovitraps containing dechlorinated tap water and water holding larvae of the same species separately. It was observed that the females of both species laid more number of eggs in ovitraps containing larval holding water than ovitraps containing distilled water. While $199.00 \pm 3.61$ eggs were laid in the larval holding water in an average by Ae. aegypti females, it was $105.00 \pm 6.00$ in distilled water. On the other hand, Ae. albopictus females laid maximum eggs in larval holding water $(208.67 \pm 8.02)$ which was $109.3 \pm 13.65$ in distilled water (Table 4).

3.5. Oviposition Preference in response to Size of Ovitrap. In order to find out if the Aedes mosquitoes have any preference for egg laying with respect to the size of the ovitrap, ovitraps of different diameters ranging from an area of $9.42 \mathrm{~cm}^{2}$ up to $50.28 \mathrm{~cm}^{2}$ containing dechlorinated tap water were used. All the ovitraps were internally coated with blotting paper halfdipped in water to provide moistened surface for oviposition.
TABLE 4: Oviposition preference in ovitraps with larva holding water by Aedes mosquitoes.

\begin{tabular}{|c|c|c|c|}
\hline \multirow{2}{*}{\multicolumn{2}{|c|}{ Types of water }} & \multicolumn{2}{|c|}{ Eggs laid by Aedes mosquitoes } \\
\hline & & Ae. aegypti & Ae. albopictus \\
\hline Distillec & ter & $105.00 \pm 6.00$ & $109.33 \pm 13.65$ \\
\hline Larval & ng water (LHW) & $199.00 \pm 3.61$ & 208.67 \\
\hline \multicolumn{4}{|c|}{$\begin{array}{l}\text { Mean } \pm \text { SD of eggs deposited in ovitraps with larva holding water by the two } \\
\text { species of Aedes mosquitoes. }\end{array}$} \\
\hline \multicolumn{4}{|c|}{$\begin{array}{l}\text { TABLE 5: Oviposition by Aedes mosquitoes in response to ovitrap } \\
\text { size. }\end{array}$} \\
\hline \multirow{2}{*}{$\begin{array}{l}\text { Serial } \\
\text { number }\end{array}$} & \multirow{2}{*}{$\begin{array}{l}\text { Surface area of ovitrap } \\
(\text { sq. } \mathrm{cm})\end{array}$} & \multicolumn{2}{|c|}{ Eggs laid by Aedes mosquitoes } \\
\hline & & Ae. aegypti & Ae. albopictus \\
\hline (1) & 9.42 & $0.00 \pm 0.00$ & $0.00 \pm 0.00$ \\
\hline (2) & 12.57 & $32.67 \pm 13.32$ & $61.67 \pm 23.71$ \\
\hline (3) & 25.14 & $92.33 \pm 15.31$ & $83.33 \pm 15.82$ \\
\hline (4) & 28.28 & $150.67 \pm 65.43$ & $120.00 \pm 12.53$ \\
\hline$(5)$ & 34.57 & $267.67 \pm 73.17$ & $208.33 \pm 53.91$ \\
\hline (6) & 37.71 & $338.00 \pm 59.43$ & $305.33 \pm 29.67$ \\
\hline$(7)$ & 50.28 & $419.67 \pm 77.42$ & $365.67 \pm 44.41$ \\
\hline
\end{tabular}

Mean number of eggs \pm S.D deposited by the two species of Aedes mosquitoes in response to ovitrap size.

The females of both species did not lay any eggs in the smallest used ovitrap. On the contrary, the maximum number of eggs was found in the largest ovitrap (Table 5). It has been observed that the number of eggs laid gradually increased with the increased size of the ovitraps exhibiting a positive correlation.

3.6. Oviposition Cycle at Different Photoperiod Regimens. The data obtained on the oviposition cycle of the two species of Aedes mosquitoes in three different light conditions, (i) tropical normal photoperiod (LD $12: 12$ ), (ii) reverse photoperiod (DL 12:12), and (iii) continuous light (24 LL), are presented in Tables 6 and 7. Observations on the number of eggs laid three hourly commencing from $6 \mathrm{pm}$ of day one to $6 \mathrm{pm}$ of day three were made to study the pattern of oviposition cycle in this species of mosquito. 
Under normal condition of tropical photoperiod (LD $12: 12$ ), the oviposition activity was maximum during 03 to $06 \mathrm{am}$ followed by 06 to $09 \mathrm{am}$ during all the three days of observation in Ae. aegypti. Negligible numbers of eggs or no eggs were laid during the other hours of observations for all the days. During the reverse photoperiod (DL 12:12) there was an unimodal oviposition cycle on all the three days between $03 \mathrm{pm}$ and $06 \mathrm{pm}$. No eggs were laid between 12 midnight and 03 am during the first 24 hours, between $09 \mathrm{pm}$ and midnight during the second 24 hours, and between $09 \mathrm{pm}$ and 03 am during the third 24 hours of observation. A maximum of $19.35 \%$ of total number of eggs were laid during 03 to $06 \mathrm{pm}$ of day one. During the same period of day two, the percentage of eggs laid was 21.85 , which was reduced to $7.92 \%$ during the same period of observation on the third day (Table 6). For studying the oviposition cycle in continuous light, the gravid blood-fed females of this species were exposed to continuous light for three days without any scotophase. Bimodal oscillations were observed during the first and second days of observation. On the day one, the maximum number of eggs was laid during $03 \mathrm{pm}$ to $06 \mathrm{pm}$. On day two and day three, the maximum number of eggs was, however, laid during $06 \mathrm{pm}$ to $09 \mathrm{pm}$. On the first day of observation, a total of 60 eggs were laid during $06 \mathrm{pm}$ to $09 \mathrm{pm}$ and on the second day 66 eggs were observed during $03 \mathrm{pm}$ to $06 \mathrm{pm}$.

Similarly, in Ae. albopictus, during the experiment with normal light-day $(12: 12)$ photoperiod, the number of eggs laid was maximum during $03 \mathrm{am}-06 \mathrm{am}$ and $06 \mathrm{am}-09 \mathrm{am}$ on all three days of observation as in the case of Ae. aegypti. During the first day of observation, the minimum number of eggs was laid between 12 noon and $03 \mathrm{pm}$. On the second day, it was between $06 \mathrm{pm}$ and $09 \mathrm{pm}$, while on the third day the minimum was between $03 \mathrm{pm}$ and $06 \mathrm{pm}$. The reverse photoperiod (DL 12:12) hinted an unimodal oviposition cycle restricted to between $03 \mathrm{pm}$ and $06 \mathrm{pm}$ on all the three days of observations. On day one while the percentage of eggs laid was 31.04 , the percentage was 19.03 and 6.59 on the second and third days, respectively. During the oviposition in continuous light two peaks were observed: the major peak between $03 \mathrm{pm}$ and $06 \mathrm{pm}$ and the minor peak between $06 \mathrm{pm}$ and $09 \mathrm{pm}$ on all the three days.

\section{Discussion}

An important aspect of mosquito ecology is to find out the factors that limit species-specific larvae to specific type of oviposition medium. Selection of oviposition site is one of the most important behavioural components of mosquito survival [2]. A proper understanding of oviposition behaviour may provide insights about mosquito life history and help in dengue surveillance and control of the vectors. Effective control of this mosquito, particularly during an outbreak of disease, can be achieved through different strategies targeting specific life-history stages.

It has been reported that ovipositing mosquitoes do not deposit eggs indiscriminately; rather their selection criteria for oviposition are probably related to the chemical nature of
TABLE 6: Oviposition cycle of Ae. aegypti in relation to different photoperiod regimens.

\begin{tabular}{|c|c|c|c|c|c|c|c|}
\hline \multirow[t]{2}{*}{ Day } & \multirow[t]{2}{*}{ Time } & \multicolumn{2}{|c|}{$\begin{array}{c}\text { Experiment-1 } \\
\text { Normal } \\
\text { (LD } 12: 12 \text { ) }\end{array}$} & \multicolumn{2}{|c|}{$\begin{array}{c}\text { Experiment-2 } \\
\text { Reverse } \\
\text { (DL } 12: 12)\end{array}$} & \multicolumn{2}{|c|}{$\begin{array}{c}\text { Experiment-3 } \\
\text { Light } \\
(24 \mathrm{LL})\end{array}$} \\
\hline & & Eggs & $\%$ & Eggs & $\%$ & Eggs & $\%$ \\
\hline \multirow{8}{*}{ One } & $18-21$ & 16 & 0.94 & 12 & 1.76 & 60 & 10.15 \\
\hline & $21-00$ & 0 & 0.00 & 6 & 0.88 & 0 & 0.00 \\
\hline & $00-03$ & 22 & 1.30 & 0 & 0.00 & 8 & 1.35 \\
\hline & $03-06$ & 334 & 19.68 & 8 & 1.17 & 18 & 3.05 \\
\hline & 06-09 & 218 & 12.85 & 12 & 1.76 & 12 & 2.03 \\
\hline & 09-12 & 0 & 0.00 & 3 & 0.44 & 0 & 0.00 \\
\hline & $12-15$ & 5 & 0.29 & 26 & 3.81 & 0 & 0.00 \\
\hline & 15-18 & 24 & 1.41 & 132 & 19.35 & 80 & 13.54 \\
\hline \multirow{8}{*}{ Two } & $18-21$ & 10 & 0.59 & 17 & 2.49 & 104 & 17.60 \\
\hline & $21-00$ & 0 & 0.00 & 0 & 0.00 & 11 & 1.86 \\
\hline & $00-03$ & 0 & 0.00 & 4 & 0.59 & 0 & 0.00 \\
\hline & $03-06$ & 350 & 20.62 & 19 & 2.79 & 34 & 5.75 \\
\hline & 06-09 & 245 & 14.44 & 48 & 7.04 & 50 & 8.46 \\
\hline & 09-12 & 12 & 0.71 & 32 & 4.69 & 14 & 2.37 \\
\hline & $12-15$ & 14 & 0.82 & 50 & 7.33 & 0 & 0.00 \\
\hline & 15-18 & 81 & 4.77 & 149 & 21.85 & 66 & 11.17 \\
\hline \multirow{9}{*}{ Three } & $18-21$ & 3 & 0.18 & 4 & 0.59 & 56 & 9.48 \\
\hline & $21-00$ & 0 & 0.00 & 0 & 0.00 & 12 & 2.03 \\
\hline & $00-03$ & 7 & 0.41 & 0 & 0.00 & 3 & 0.51 \\
\hline & $03-06$ & 210 & 12.37 & 11 & 1.61 & 32 & 5.41 \\
\hline & 06-09 & 111 & 6.54 & 38 & 5.57 & 21 & 3.55 \\
\hline & 09-12 & 21 & 1.24 & 13 & 1.91 & 10 & 1.69 \\
\hline & $12-15$ & 9 & 0.53 & 44 & 6.45 & 0 & 0.00 \\
\hline & 15-18 & 5 & 0.29 & 54 & 7.92 & 0 & 0.00 \\
\hline & Total & 1697 & & 682 & & 591 & \\
\hline
\end{tabular}

Oviposition cycle of Ae. aegypti in relation to normal (LD $12: 12$ ), reverse (DL $12: 12$ ), and continuous light (24 LL) photoperiods.

the water. Different species of mosquitoes have been classified distinctly on the basis of their preference for salt water or fresh water for oviposition. According to Wallis [9], larvae of many mosquito species develop well in salt water and, therefore, such species prefer higher salt content of the medium for oviposition. In the present investigation, the authors were inclined to study the oviposition response of the ovipositing females belonging to the two species of Aedes mosquitoes to oviposition media containing different concentrations of sodium chloride.

Wallis [12] has shown that $0.5 \% \mathrm{NaCl}$ solution was preferred over distilled water for oviposition by these mosquitoes. In the present experiments, although the maximum number of eggs was laid by Ae. aegypti females in $0.25 \%$ $\mathrm{NaCl}$ concentration, Ae. albopictus females laid the maximum number of eggs in distilled water. In both the mosquito species the number of eggs laid gradually decreased with an increase in $\mathrm{NaCl}$ concentration in the oviposition medium which is almost similar to the findings of Macfie [13] wherein he has reported that Aedes mosquitoes do not lay eggs in 
TABLE 7: Oviposition cycle of Ae. albopictus in relation to different photoperiod regimens.

\begin{tabular}{|c|c|c|c|c|c|c|c|}
\hline \multirow[t]{2}{*}{ Day } & \multirow[t]{2}{*}{ Time } & \multicolumn{2}{|c|}{$\begin{array}{l}\text { Experiment-1 } \\
\text { Normal } \\
(\text { LD } 12: 12)\end{array}$} & \multicolumn{2}{|c|}{$\begin{array}{c}\text { Experiment-2 } \\
\text { Reverse } \\
\text { (DL } 12: 12)\end{array}$} & \multicolumn{2}{|c|}{$\begin{array}{c}\text { Experiment-3 } \\
\text { Light } \\
(24 \mathrm{LL})\end{array}$} \\
\hline & & Eggs & $\%$ & Eggs & $\%$ & Eggs & $\%$ \\
\hline \multirow{8}{*}{ One } & $18-21$ & 19 & 1.16 & 22 & 3.22 & 42 & 11.67 \\
\hline & $21-00$ & 11 & 0.67 & 3 & 0.44 & 0 & 0.00 \\
\hline & $00-03$ & 62 & 3.77 & 0 & 0.00 & 0 & 0.00 \\
\hline & $03-06$ & 285 & 17.35 & 0 & 0.00 & 12 & 3.33 \\
\hline & 06-09 & 270 & 16.43 & 36 & 5.27 & 16 & 4.44 \\
\hline & 09-12 & 18 & 1.10 & 17 & 2.49 & 4 & 1.11 \\
\hline & $12-15$ & 10 & 0.61 & 64 & 9.37 & 0 & 0.00 \\
\hline & 15-18 & 48 & 2.92 & 212 & 31.04 & 61 & 16.94 \\
\hline \multirow{8}{*}{ Two } & $18-21$ & 8 & 0.49 & 10 & 1.46 & 34 & 9.44 \\
\hline & $21-00$ & 21 & 1.28 & 4 & 0.59 & 4 & 1.11 \\
\hline & $00-03$ & 31 & 1.89 & 0 & 0.00 & 3 & 0.83 \\
\hline & $03-06$ & 256 & 15.58 & 2 & 0.29 & 18 & 5.00 \\
\hline & 06-09 & 218 & 13.27 & 21 & 3.07 & 20 & 5.56 \\
\hline & 09-12 & 26 & 1.58 & 14 & 2.05 & 0 & 0.00 \\
\hline & $12-15$ & 16 & 0.97 & 52 & 7.61 & 0 & 0.00 \\
\hline & $15-18$ & 50 & 3.04 & 130 & 19.03 & 56 & 15.56 \\
\hline \multirow{9}{*}{ Three } & $18-21$ & 3 & 0.18 & 7 & 1.02 & 31 & 8.61 \\
\hline & $21-00$ & 11 & 0.67 & 2 & 0.29 & 0 & 0.00 \\
\hline & $00-03$ & 9 & 0.55 & 0 & 0.00 & 0 & 0.00 \\
\hline & $03-06$ & 144 & 8.76 & 0 & 0.00 & 12 & 3.33 \\
\hline & 06-09 & 99 & 6.03 & 9 & 1.32 & 13 & 3.61 \\
\hline & 09-12 & 24 & 1.46 & 10 & 1.46 & 0 & 0.00 \\
\hline & $12-15$ & 3 & 0.18 & 23 & 3.37 & 0 & 0.00 \\
\hline & $15-18$ & 1 & 0.06 & 45 & 6.59 & 34 & 9.44 \\
\hline & Total & 1643 & & 683 & & 360 & \\
\hline
\end{tabular}

Oviposition cycle of Ae. albopictus in relation to normal (LD 12:12), reverse (DL 12 : 12), and continuous light (24 LL) photoperiods.

sea water. However, according to Woodhill [14], eggs were still laid in diluted sea water containing up to $1.75 \% \mathrm{NaCl}$. It is difficult to understand the oviposition behaviour with respect concentration of $\mathrm{NaCl}$ in the oviposition medium. This could be probably because the ovipositing females lack choice for laying eggs or, alternately, a low concentration of the salt could be providing nutrients for larval sustenance and growth. From the present findings, it may be concluded that Aedes mosquitoes have a wider range of preference for salinity of the oviposition medium for egg laying.

According to Reiskind and Zarrabi [8], Aedes mosquitoes are day active mosquitoes and, therefore, might be relying more on optical cues like the contrast between dark container openings and water surface (specular) reflections for selection of resting and oviposition sites than night active mosquito species. In order to find out if ovipositing Aedes mosquitoes have any choice for the colour of the medium for egg laying, it was decided to use six different coloured ovistrips to study their oviposition behaviour. It was recorded that the maximum number of eggs was laid by the females of both species on black ovistrip followed by red ovistrip. However, there was no statistical difference between the number of eggs laid by them on black and red ovistrips. This may lead us to conclude that the Aedes mosquitoes cannot differentiate between these two colours. Similar observations were made in different species of mosquitoes by Williams [15], Wilton [16], Mc Daniel et al. [17], Beehler et al. [18], Jones and Schreiber [19], and Yanoviak [20]. According to Chapman [21], most insects are unable to see red; therefore, the red ovistrips probably appear dark grey to the mosquitoes. Colton et al. [4] have however reported that the Aedes mosquitoes lay maximum number of eggs on black ovistrips. Again, Aedes mosquitoes breed in different types of household containers including flower pots. In the study area people use different coloured pots, especially yellow and green ones, for planting flowering plants. Therefore, the authors were interested to find out the preference for such coloured ovistrips for oviposition by the Aedes females. Yellow and green colours are probably not distinguished by the Aedes mosquitoes; as a result they have laid almost similar number of eggs on the ovistrips of these two colours. White ovistrips were least preferred for oviposition by the Aedes mosquitoes which confirm the earlier report of Chua et al. [22]. Since the number of eggs laid in dark or lighted condition on different colour ovistrips did not vary significantly except for white ovistrips on which no eggs were laid by either of the species in dark, it may be concluded that the Aedes mosquitoes have either no or very little visual perception.

The choice of an appropriate oviposition site has a great influence on maternal reproductive success in species with aquatic larval and pupae stages [23]. This is because several attributes of the water, both physical and chemical, influence hatching success and larval survival [24]. Therefore, the mosquitoes have a strong selection for discrimination of potential oviposition sites based on offspring viability [25]. Gravid females follow visual or olfactory cues to appropriate water collections and are guided by chemical cues and physical factors in the water and the quality of water before deciding to lay their eggs $[26,27]$. Our findings demonstrate that both of these species of mosquitoes prefer clear and clean water Mc Daniel et al. as compared to polluted water.

It has been reported that ovipositing females normally choose water with the presence of life $[2,28]$. Mosquitoes normally avoid oviposition where interspecific competitors are present [3] but are attracted to sites where other mosquito larvae are present [29] because the presence of conspecific larvae may provide a reliable cue that the site offers suitable conditions for larval development [30]. In the present experiment too, the gravid females of both species preferred larval holding water over distilled water.

In the present study, it was found that the ovipositing females prefer larger surface area of ovitrap than the smaller ones. The number of eggs laid has a distinct bearing on the area of the ovitrap exhibiting a direct linear relationship between the surface area and the number of eggs laid which was similar to the observations made by Derraik and Slaney [31]. It is believed that the larger containers may provide large, protected, humid resting surfaces for females preparing for oviposition [8]. Further, in larger containers, the chances of 
desiccation are less and are likely to contain more amount of food and thus are likely to increase the chances of larval survival. Thus it may be concluded that Aedes mosquitoes prefer large sized containers holding water for oviposition compared to the ones with smaller dimensions.

Oviposition rhythms have been reported to be different in different species of mosquitoes [32-38]. Further, the oviposition time is considered to be the interplay between intrinsic and extrinsic factors. The intrinsic factors include the release of ovarian development hormone and ovulation hormone which may be associated with the time of blood meal and mating in Aedes mosquitoes [39-41]. The extrinsic factor appears to be light for the determination of time of oviposition. In the present experiments with normal $12 \mathrm{~h}$ light and dark phases (LD 12:12), females of both Aedes species laid the maximum number of eggs in the 4 th quarter of the light period which gradually reduced thereafter on all the three days of the experiment. Our results differ from those of Panicker et al. [38] but agree with the observations of Haddow and Gillett [34], Christophers [42], Gillett et al. [35, 43], and Chand [44]. In the experiments with reverse photoperiod, similar observations were made for both species. The peak egg laying was mostly during the 3rd quarter of the light phase that is during the 4 th quarter of the original light phase. This probably reflects the influence of the intrinsic factors on egg laying which has not been altered due to artificial alternation of photoperiod. In the experiment with continuous light (24 LL) there was irregularity in oviposition, which indicated that light phase is essential for normal oviposition by the Aedes females. Thus the present finding clearly indicates that light (duration) acts as the most important factor in regulating the intrinsic factors responsible for cyclical oviposition in Aedes mosquitoes.

Till now, Aedes mosquito control is the only option to stop dengue virus transmission. Understanding various factors that favour mosquito population abundance is paramount for implementing successful mosquito vector control programs. In the present investigation, we used wild Aedes mosquitoes collected from different localities in and around Berhampur city. We observed that female mosquitoes of both species laid the maximum number of eggs in the 4 th quarter of the light period. They usually preferred to lay eggs in ovitraps containing distilled water only as compared to other water collected from different sources. Further, Aedes females laid more number of eggs in ovitraps containing larval holding water. Usually theses mosquitoes avoid very small containers for oviposition and preferred black colour ovistrip for laying eggs. Probably $\mathrm{NaCl}$ is not favourable for the oviposition and it was found that the number of eggs laid gradually decreased with an increase in the concentration of $\mathrm{NaCl}$ in the oviposition medium in both the species. Our recommendation here is that, based on these findings, control tools and strategies could be possible to design and develop suitable lethal ovitrap for the control of these mosquito vectors.

\section{Conflict of Interests}

The authors declare that they have no conflict of interests regarding the publication of this paper.

\section{References}

[1] D. J. Gubler, "The global emergence/resurgence of arboviral diseases as public health problems," Archives of Medical Research, vol. 33, no. 4, pp. 330-342, 2002.

[2] M. D. Bentley and J. F. Day, "Chemical ecology and behavioral aspects of mosquito oviposition," Annual Review of Entomology, vol. 34, pp. 401-421, 1989.

[3] L. Blaustein and B. P. Kotler, "Oviposition habitat selection by the mosquito, Culiseta longiareolata: effects of conspecifics, food and green toad tadpoles," Ecological Entomology, vol. 18, no. 2, pp. 104-108, 1993.

[4] Y. M. Colton, D. D. Chadee, and D. W. Severson, "Natural "skip oviposition" of the mosquito Aedes aegypti as evidenced by codominant genetic markers," Medical and Veterinary Entomology, vol. 17, no. 2, pp. 195-201, 2003.

[5] U. D. Kitron, D. W. Webb, and R. J. Novak, "Oviposition behavior of Aedes triseriatus (Diptera: Culicidae): prevalence, intensity, and aggregation of eggs in oviposition traps," Journal of Medical Entomology, vol. 26, no. 5, pp. 462-467, 1989.

[6] D. D. Chadee, P. S. Corbet, and J. J. D. Greenwood, "Egg-laying Yellow Fever Mosquitoes avoid sites containing eggs laid by themselves or by conspecifics," Entomologia Experimentalis et Applicata, vol. 57, no. 3, pp. 295-298, 1990.

[7] B. L. Apostol, W. C. Black, P. Reiter, and B. R. Miller, "Use of randomly amplified polymorphic DNA amplified by polymerase chain reaction markers to estimate the number of Aedes aegypti families at oviposition sites in San Juan, Puerto Rico," The American Journal of Tropical Medicine and Hygiene, vol. 51, no. 1, pp. 89-97, 1994.

[8] M. H. Reiskind and A. A. Zarrabi, "Water surface area and depth determine oviposition choice in Aedes albopictus (Diptera: Culicidae)," Journal of Medical Entomology, vol. 49, no. 1, pp. 7176, 2012.

[9] R. C. Wallis, "A study of the oviposition activity of three species of Anopheles in the laboratory," American Journal Medicine, vol. 4, pp. 557-563, 1955.

[10] M. Suleman and M. Shirin, "Laboratory studies on oviposition behaviour of Culex quinquefasciatus Say (Diptera: Culicidae): choice of oviposition medium and oviposition cycle," Bulletin of Entomological Research, vol. 71, pp. 361-369, 1981.

[11] S. D. Beck, Insect Photoperiodism, Academic Press, New York, NY, USA, 1968.

[12] R. C. Wallis, "The effect of population density and of $\mathrm{NaCl}$ concentrations in test series in laboratory experiments with ovipositing Aedes aegypti," Mosquito News, vol. 14, pp. 200-204, 1954.

[13] J. W. S. Macfie, "Effect of saline solutions and sea water on Stegomyia fasciata," Annals of Tropical Medicine and Parasitology, vol. 45, pp. 137-140, 1921.

[14] A. R. Woodhill, "The oviposition responses of three species of mosquitoes Aedes (Stegomyia) aegypti (Linn., Culex (culex) fatigans Wiedemann, Aedes (Pseudskusea) concolor Taylor, in relation to the salinity of the water," Proceedings of the Linnean Society of New South Wales, vol. 66, pp. 287-292, 1941.

[15] R. E. Williams, "Effect of coloring oviposition media with regard to the mosquito Aedes triseriatus (Say)," Journal of Parasitology, vol. 48, pp. 919-925, 1962.

[16] D. P. Wilton, "Oviposition site selection by the tree-hole mosquito, Aedes triseriatus (Say)," Journal of Medical Entomology, vol. 5, no. 2, pp. 189-194, 1968. 
[17] I. N. McDaniel, M. D. Dentley, H. P. Lee, and M. Yatagai, "Effects of color and larval-produced oviposition attractants on oviposition of Aedes triseriatus," Environmental Entomology, vol. 5, pp. 553-556, 1976.

[18] J. Beehler, S. Lohr, and G. DeFoliart, "Factors influencing oviposition in Aedes triseriatus Diptera: culicidae," Great Lakes Entomologist, vol. 25, no. 4, pp. 259-264, 1992.

[19] C. J. Jones and E. T. Schreiber, "Color and height affects oviposition site preferences of Toxorhynchites splendens and Toxorhynchites rutilus rutilus (Diptera: Culicidae) in the laboratory," Environmental Entomology, vol. 23, no. 1, pp. 130-135, 1994.

[20] S. P. Yanoviak, "Container color and location affect macroinvertebrate community structure in artificial treeholes in Panama," Florida Entomologist, vol. 84, no. 2, pp. 265-271, 2001.

[21] R. F. Chapman, The Insects: Structure and Function, Cambridge University Press, Cambridge, UK, 4th edition, 1998.

[22] K. B. Chua, I.-.E. Chua, I.-.L. Chua, and K. H. Chua, "Differential preferences of oviposition by Aedes mosquitos in man-made containers under field conditions," Southeast Asian Journal of Tropical Medicine and Public Health, vol. 35, no. 3, pp. 599-607, 2004.

[23] J. G. Millar, J. D. Chaney, J. W. Beehler, and M. S. Mulla, "Interaction of the Culex quinquefasciatus egg raft pheromone with a natural chemical associated with oviposition sites," Journal of the American Mosquito Control Association, vol. 10, no. 3, pp. 374-379, 1994.

[24] W. J. Resetarits Jr. and H. M. Wilbur, "Choice of oviposition site by Hyla chrysoscelis: role of predators and competitors," Ecology, vol. 70, no. 1, pp. 220-228, 1989.

[25] J. W. Petranka and K. Fakhourry, "Evidence of a chemically mediated response of ovipositing insects to bluegills and green frog tadpoles," Copeia, vol. 19, pp. 273-279, 1991.

[26] D. D. Bonnets and H. Chapman, "The importance of mosquito breeding tree holes, with special reference to the problems in Tahiti," Mosquito News, vol. 16, pp. 301-305, 1956.

[27] D. A. Muir, "Anopheline mosquitoes: nector reproduction, life-cycle and biotype," in Malaria, Principles and Practice of Malariology, H. W. Wernsdorfer and J. McGregor, Eds., pp. 431451, Churchill-Livingstone, London, UK, 1988.

[28] S. J. Lee, "Major factors affecting mosquito oviposition," Chinese Journal of Entomology, vol. 6, pp. 23-35, 1991.

[29] J. W. Beehler and M. S. Mulla, "Effects of organic enrichment on temporal distribution and abundance of culicine egg rafts," Journal of the American Mosquito Control Association, vol. 11, no. 2, pp. 167-171, 1995.

[30] G. Stay, L. Blaustein, and J. Margalith, "Experimental evidence for predation risk sensitive oviposition by a mosquito, Culiseta longiareolata," Ecological Entomology, vol. 24, no. 2, pp. 202-207, 1999.

[31] J. G. B. Derraik and D. Slaney, "Container aperture size and nutrient preferences of mosquitoes (Diptera: Culicidae) in the Auckland region, New Zealand," Journal of Vector Ecology, vol. 30, no. 1, pp. 73-82, 2005.

[32] T. R. C. Muirhead, "Studies on the behaviour of Anopheles minimus - part I: the selection of the breeding place and the influence of light and shade," Journal of Malaria Institute of India, vol. 3, p. 265, 1940.

[33] J. D. Gillett, "Notes on the subgenus Coquillettidia Dyar (Diptera, Culicidae)," Bulletin of Entomological Research, vol. 36, pp. 425-438, 1946.
[34] A. J. Haddow and J. D. Gillett, "Observations on the oviposition -cycle of Aedes ( Stegomyia ) aegypti (Linn.)," Annals of Tropical Medicine and Parasitology, vol. 51, no. 2, pp. 159-169, 1957.

[35] J. D. Gillett, P. S. Corbet, and A. J. Haddow, "Observations on the oviposition-cycle of Aedes (Stegomyia) aegypti (Linnaeus)," Annals of Tropical Medicine and Parasitology, vol. 53, pp. 132136, 1959.

[36] T. Yajima, "Ecological studies on the gonadotrophic cycle of the mosquito, Culex tritaeniorhynchus summorosus Dyar, in relation to the ovarian condition," Science Reports of the Tohoku University 4: Biology, vol. 36, no. 4, pp. 241-253, 1973.

[37] A. Q. Khan and W. K. Reisen, "Laboratory observations on developmental rhythms in Culex tritaeniorhynchus," Mosquito News, vol. 37, no. 4, pp. 637-645, 1977.

[38] K. N. Panicker, M. G. Bai, and K. Viswam, "Observation on the oviposition rhythm of some mosquitoes," The Indian Journal of Medical Research, vol. 77, pp. 388-391, 1981.

[39] J. D. Gillett, "Behaviour differences in two strains of Aëdes aegypti," Nature, vol. 176, no. 4472, pp. 124-125, 1955.

[40] J. D. Gillett, "Genetic differences affecting egg-laying in the mosquito Aedes (Stegomyia) aegypti (Linn)," Annals of Medicine, vol. 50, p. 362, 1956.

[41] J. D. Gillett, "Initiation and promotion of ovarian development in the mosquito Aedes (Stegomyia) aegypti (Linn.)," Annals of Tropical Medicine and Parasitology, vol. 50, p. 375, 1956.

[42] S. R. Christophers, Aedes aegypti L. The Yellow Fever Mosquito. Its Life History, Bionomics and Structure, Cambridge University Press, Cambridge, UK, 1960.

[43] J. D. Gillett, P. S. Corbet, and A. J. Haddow, "Observations on the oviposition cycle of Aedes aegypti," Annals of Tropical Medicine and Parasitology, vol. 55, pp. 427-431, 1961.

[44] S. K. Chand, Ecology and population dynamics of certain species of mosquitoes [Ph.D. thesis], Berhampur University, 1989. 

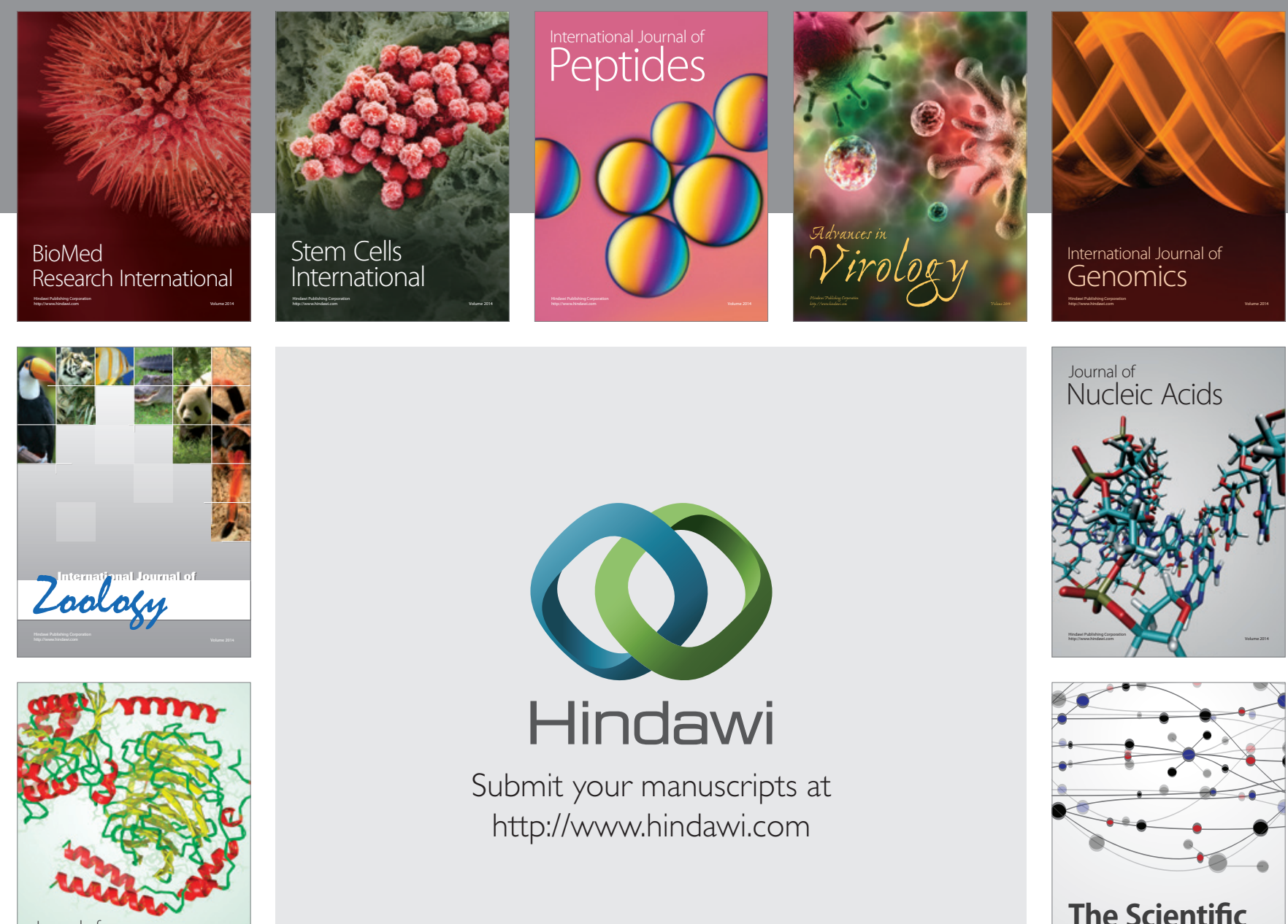

Submit your manuscripts at

http://www.hindawi.com

Journal of
Signal Transduction
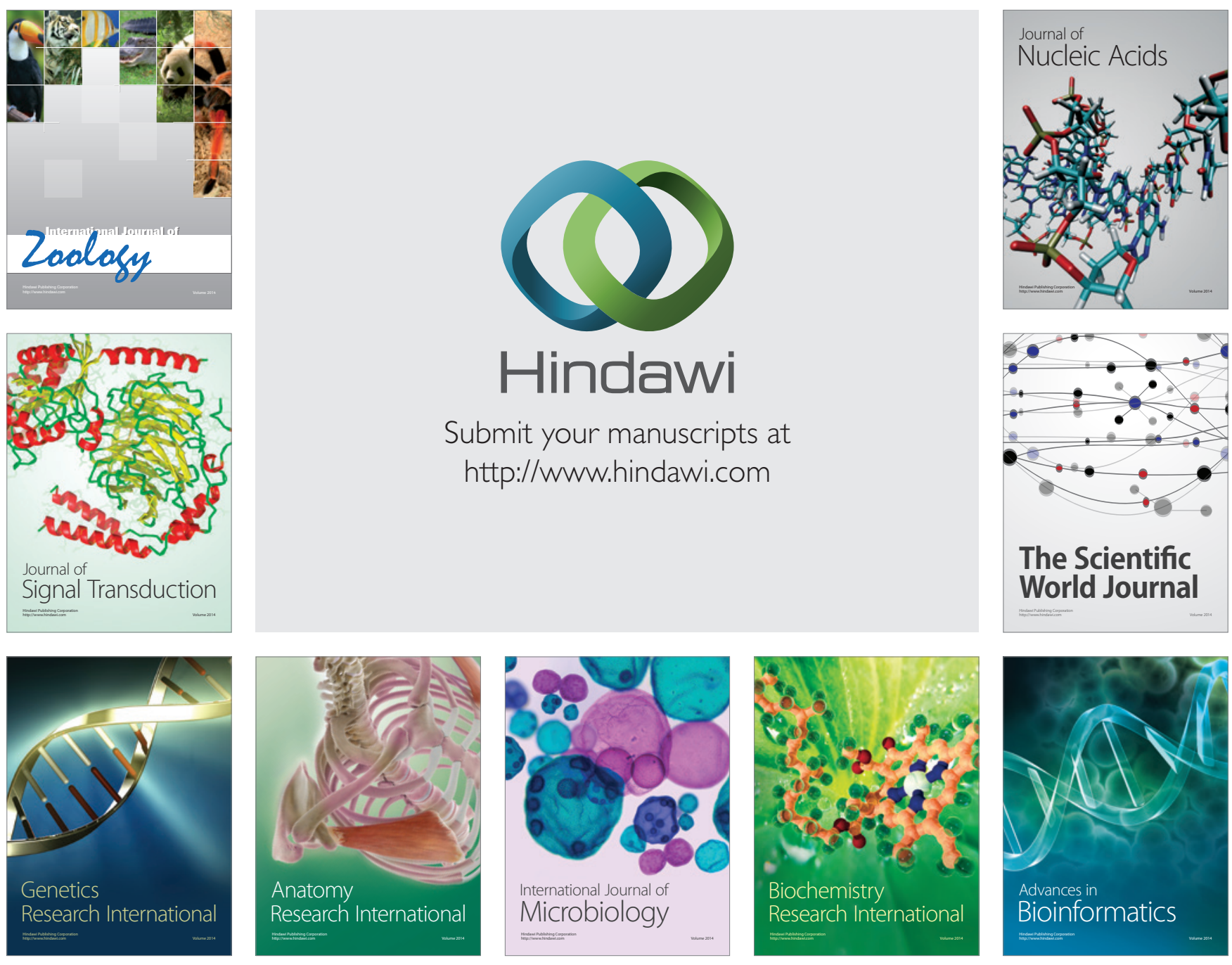

The Scientific World Journal
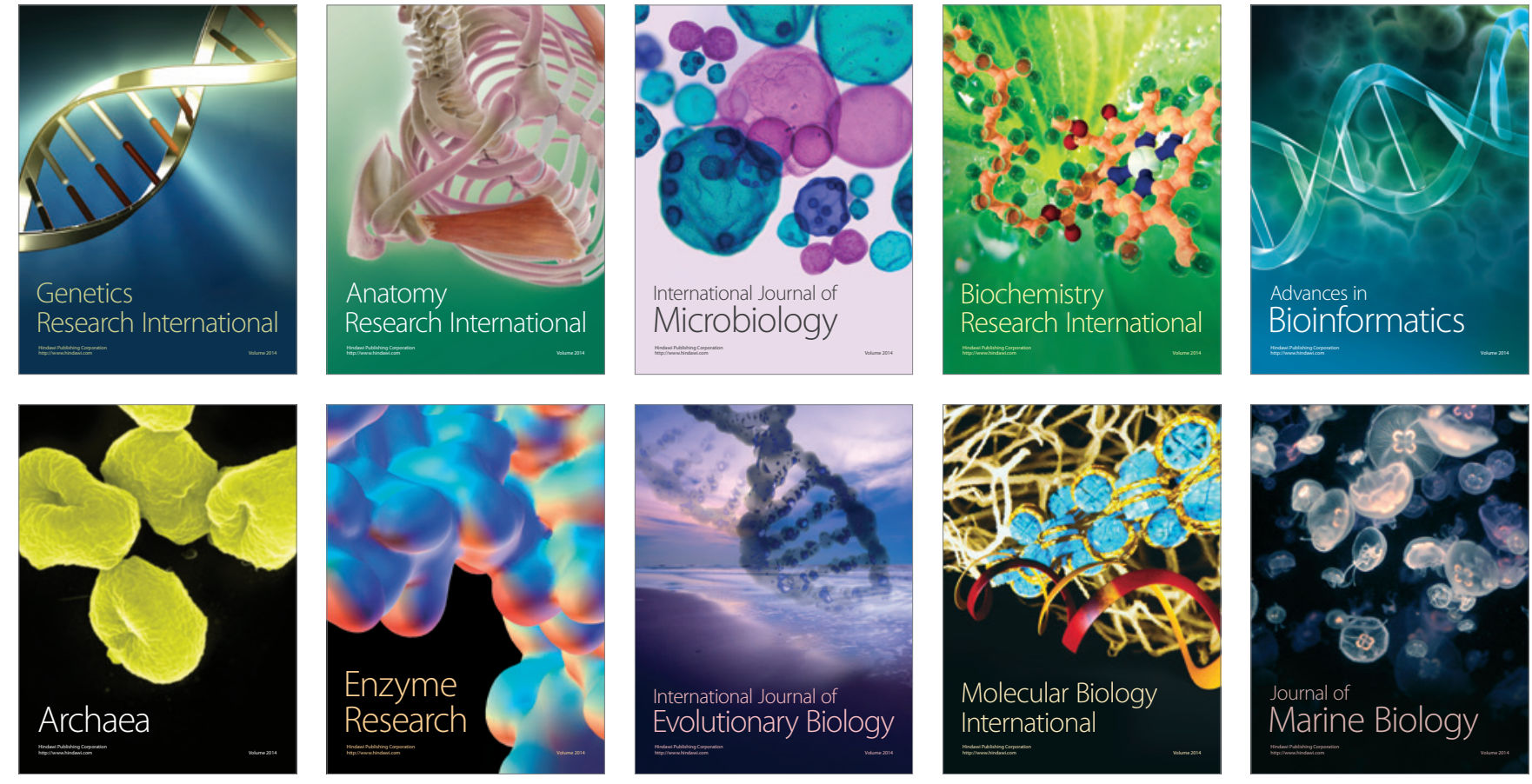